\title{
Sedentary work and the risk of breast cancer in pre-and post-menopausal women: A pooled analysis of two case-control studies
}

Terry Boyle ${ }^{1,2,3,4}$, Lin Fritschi ${ }^{4}$, Lindsay C. Kobayashi ${ }^{5,6,7}$, Jane S. Heyworth ${ }^{8}$, Derrick G. Lee $^{1,2}, \mathrm{Si} \mathrm{Si}^{4}$, Kristan J. Aronson ${ }^{6,7}$, John J. Spinelli ${ }^{1}$

1. Cancer Control Research, British Columbia Cancer Agency, Vancouver, British Columbia, Canada

2. School of Population and Public Health, The University of British Columbia, Vancouver, British Columbia, Canada

3. Centre for Medical Research, The University of Western Australia, Perth, Western Australia, Australia

4. School of Public Health, Curtin University, Perth, Western Australia, Australia

5. Department of Epidemiology and Public Health, University College London, London, United Kingdom

6. Department of Public Health Sciences, Queen's University, Kingston, Canada

7. Division of Cancer Care and Epidemiology, Cancer Research Institute, Queen's University, Kingston, Canada

8. School of Population Health, The University of Western Australia, Perth, Western Australia, Australia

\section{Corresponding Author}

Dr. Terry Boyle

School of Public Health

Curtin University

GPO Box U1987

PERTH WA 6845 AUSTRALIA

Tel: $\quad$ (+ 61) 892662753

E-mail: terry.boyle@curtin.edu.au

Keywords: sedentary work; breast cancer; job-exposure matrix

Word Count: 3677 


\section{ABSTRACT}

Objectives: There is limited research on the association between sedentary behaviour and breast cancer risk, particularly whether sedentary behaviour is differentially associated with pre- and post-menopausal breast cancer. We pooled data from two case-control studies from Australia and Canada to investigate this association.

Methods: This pooled analysis included 1762 incident breast cancer cases and 2532 controls. Participants in both studies completed a lifetime occupational history and self-rated occupational physical activity level. A job-exposure matrix (JEM) was also applied to job titles to assess sedentary work. Logistic regression analyses (six pooled and twelve studyspecific) were conducted to estimate associations between both self-reported and JEMassessed sedentary work and breast cancer risk among pre- and post-menopausal women.

Results: No association was observed in the six pooled analyses, and ten of the studyspecific analyses also showed null results. Two study-specific analyses provided inconsistent and contradictory results, with one showing statistically significant increased risk of breast cancer for self-reported sedentary work among pre-menopausal women cancer in the Canadian study, and the other a non-significant inverse association between JEM-assessed sedentary work and breast cancer risk among post-menopausal women in the Australian study.

Conclusions: While a suggestion of increased risk was seen for pre-menopausal women in the Canadian study when using the self-reported measure, overall this pooled study does not provide evidence that sedentary work is associated with breast cancer risk. 


\section{What this paper adds}

There is research interest in the association between sedentary behaviour and breast cancer risk, particularly whether sedentary behaviour is differentially associated with pre- and post-menopausal breast cancer.

This pooled analysis of two population-based case-control studies did not find strong evidence that sedentary work is associated with an increased risk of breast cancer.

Occupational sedentary behaviour may not have a large influence on overall breast cancer risk. 


\section{INTRODUCTION}

Approximately 100 studies conducted during the past 20 to 30 years provide convincing evidence that increased levels of moderate-to-vigorous intensity physical activity are associated with lower breast cancer risk [1]. Far fewer studies have investigated the association between breast cancer risk and sedentary behaviour (activities performed in a seated or lying position during waking hours that require very little energy expenditure [2]). Recent research suggests that sedentary behaviour may be associated with higher mortality and increased risks of diabetes, cardiovascular disease, and colon, endometrial and lung cancers $[3,4]$, independent of physical activity, with associations generally observed among people with high and low physical activity levels [3]. Among working adults, the majority of sedentary behaviour is performed in the occupational domain [5], so it is important to investigate how sedentary behaviour at work is associated with the risk of breast cancer, which is by far the most common cancer among females in both less developed and more developed world regions [6]. The workplace is also an important setting for health promotion [7] and may represent an ideal site to intervene to reduce sedentary behaviour.

Sedentary behaviour is associated with central adiposity, insulin resistance and inflammation, all of which are thought to be involved in breast cancer carcinogenesis [8]; therefore, it is biologically plausible that sedentary behaviour may increase the risk of breast cancer. However, research investigating sedentary behaviour and breast cancer risk has produced inconsistent results. Two meta-analyses of 21 cohort and case-control studies and three cohort studies, respectively, found that females with the highest levels of sedentary behaviour have a slightly higher risk of breast cancer compared to females with the lowest sedentary levels [9, 10]. A third meta-analysis of 13 cohort and case-control studies concluded there was no association between sedentary behaviour and breast cancer risk [4]. 
Some of this inconsistency may be because few studies have accounted for menopausal status. Although menopausal status influences the associations between breast cancer risk, physical activity, and obesity - two risk factors related to sedentary behaviour [11] - only six studies have examined whether sedentary behaviour may be differentially associated with the risks of pre-menopausal and post-menopausal breast cancer [12-17].

We pooled data from two population-based case-control studies in Australia and Canada to investigate the associations between sedentary behaviour at work and breast cancer risk among pre-menopausal women and post-menopausal women. Pooling the two studies, rather than simply analysing each study individually, gave us the ability to examine associations with sedentary behaviour among pre-menopausal women and post-menopausal women separately, and to investigate potential effect modification by lifetime recreational physical activity. Both studies have previously reported inverse associations between moderate-to-vigorous intensity physical activity and the risk of breast cancer, with larger risk reductions found among postmenopausal women compared to premenopausal women [1820]. This is the first analysis of sedentary behaviour in these studies.

\section{METHODS}

The participants in this pooled analysis were from the Breast Cancer Environment and Employment Study (BCEES) and the Canadian Breast Cancer Study (CBCS) [19, 21]. This analysis only included cases with invasive breast cancer (non-invasive breast cancer cases were recruited in the CBCS but not the BCEES) and participants aged 40 years and older (younger participants were recruited in the BCEES but not in the CBCS). The BCEES was conducted in Western Australia (WA) between 2009 and 2011, and recruited 1123 incident (invasive) breast cancer cases (58\% response) and 1705 age-matched controls (randomly selected from the WA electoral roll; 41\% response) aged 40 years and older. Cases were 
identified and recruited through the population-based WA Cancer Registry, based on mandatory reporting of invasive cancer by pathology laboratories and other clinical sites. The CBCS was conducted in Vancouver, British Columbia (BC) and Kingston, Ontario between 2006 and 2010, and recruited 913 incident (invasive) breast cancer cases (identified and recruited from the BC Cancer Registry and the Hotel Dieu Hospital Breast Assessment Program in Kingston, Ontario; 54\% and 59\% response, respectively) and 1169 age-matched controls (randomly selected cancer-free women enrolled in the Screening Mammography Program of BC; and, cancer-free women attending the Hotel Dieu Hospital Breast Assessment Program in Ontario; 57\% and 49\% response, respectively) aged 40 years and older. Both studies were approved by human research ethics committees and informed consent was obtained from all participants.

Participants in both studies completed questionnaires (either self-administered or via telephone interview) about demographic factors, education, family history of breast cancer, medical and reproductive history, menopausal status, anthropometrics, tobacco and alcohol use, lifetime physical activity, and lifetime occupational history including physical activity on the job. The classification of the variables included in this analysis is outlined for each study in Supplementary Table 1. Participants with missing occupation data (15 cases and 15 controls in BCEES, 34 cases and 30 controls in CBCS) or missing covariate data (168 cases and 238 controls in BCEES, 57 cases and 59 controls in CBCS) were excluded, leaving 4294 participants in this pooled analysis (Total: 1762 cases, 2532 controls; BCEES: 940 cases, 1452 controls; CBCS: 822 cases, 1080 controls).

Menopausal Status

In BCEES, participants were classified as post-menopausal if they reported that they were no longer having regular menstrual periods because of menopause. Additionally, 
participants aged 51 years or older were classified as being post-menopausal if: they reported that they no longer had a regular menstrual period because of cancer treatment, hysterectomy or oophorectomy; they reported that they had irregular menstrual periods possibly because of menopause; they reported that they had regular menstrual periods and were taking hormone replacement therapy; or they were missing information on whether they had regular menstrual periods. In CBCS, participants were classified as post-menopausal if they: reported natural cessation of menstrual periods at least 12 months ago; reported natural cessation of menstruation and were older than 50 years of age (if time since last menstruation was missing); or had undergone a bilateral oophorectomy. In addition, participants aged 55 years or older were classified as being post-menopausal if they reported that their menstrual periods stopped due to other reasons (e.g. cancer treatment).

Exposure Measurement

In both studies, for each job (defined as paid employment, volunteer positions and post-secondary education) held for more than six months, participants were asked to record job title and tasks, industry, calendar years started and ended, the number of hours per week worked, and the level of occupational activity required.

In the BCEES, participants could choose from the following occupational activity categories: sedentary, standing, manual, or heavy manual [22]. In the CBCS, participants could choose sedentary, light, moderate, or heavy [23]. In the BCEES questionnaire, a sedentary occupation was defined as a job in which "you spend most of your time sitting (such as in an office)", while a standing occupation was defined as a job in which "you spend most of your time standing and walking, however your work does not require intense physical effort (e.g., shop assistant, hairdresser, guard)" (Supplementary Figure 1). In the CBCS questionnaire, a sedentary occupation was defined as “work that involves sitting only, with 
minimal walking”, while a light occupation was defined as “work that involves minimal physical effort such as standing and slow walking with no increase in heart rate and perspiration” (Supplementary Figure 1). The occupational physical activity questionnaires used in both studies have acceptable test-retest reliability [22, 23]. The manual and heavy manual categories in BCEES and moderate and heavy categories in CBCS were combined into one 'active' category'. Jobs with missing occupational activity level were assigned either the level of a similar job the same participant had held, or a physical demands strength rating (based on job-title and duties) from the Dictionary of Occupational Titles if the participant had not held or had not reported the activity level of a similar job [24, 25].

We also used two job-exposure matrices (JEMs) to assess occupational physical activity, both of which are based on Occupational Information Network (O*NET) data [26, 27]. The O*NET 'Work Context - Spend Time Sitting’ index was used to assess sedentary behaviour at work (hereafter referred to as Sit-JEM). The second JEM, the O*NET 'Work Context - Spend Time Walking or Running’ index (hereafter referred to as Walk-JEM), was used only to differentiate between standing and 'active' jobs. A third JEM, the O*NET 'Work Context - Spend Time Standing' index (hereafter referred to as Stand-JEM), was used when comparing self-reported and JEM-assessed occupational activity levels but was not used to assess occupational physical activity. In all three JEMs, O*NET assigns a numerical value (from 0 to 100) to 942 different occupations (i.e., 942 individual United States Standard Occupational Classification 2010 codes) based on how much of the particular activity is required. These values are derived from questionnaires completed by job incumbents, occupational experts and/or occupational analysts, who categorize each specific job as requiring the activity (e.g., sitting) to be performed none of the time, less than half the time, about half the time, more than half the time, or continually or almost continually. Values of 0 , 
25, 50, 75 and 100 are then assigned to the respective categories, and the final value used in the $\mathrm{O}^{*}$ NET database for each specific job is the mean of these assigned values.

We used two algorithms to categorise JEM-assessed occupational physical activity. In the first (Sit-JEM75), jobs with a Sit-JEM value of 75 or higher were classified as sedentary jobs (representing jobs that require sitting more than half the time), jobs with a Walk-JEM value of 50 or higher were classified as active jobs, and all other jobs were classified as standing jobs. In the second (Sit-JEM90), a more conservative (although arbitrary) definition of sedentary jobs was used: a Sit-JEM value of 90 or higher. Jobs with a Walk-JEM value of 50 or higher were classified as active jobs, and all other jobs (including jobs with a Sit-JEM value between 75 and 90) were classified as standing jobs.

In both studies we calculated the number of full-time equivalent years (assuming a full time job is 40 hours per week for 48 weeks per year) each participant had spent in sedentary, standing and active work for self-reported and JEM-assessed activity. Full-time equivalent years spent in sedentary occupations was categorised into four groups: 0 years, and three groups of equal size (tertiles) based on the distribution among controls who had ever worked in a sedentary occupation.

Statistical Analysis

Logistic regression was used to estimate the association between sedentary work and the risk of breast cancer. Age and study site (Western Australia; British Columbia or Ontario) were included as covariates in all analyses. A causal diagram (directed acyclic graph) was used to select a sufficient set of confounders (i.e., a set of confounders that 'blocked' all backdoor (confounding) pathways, based on the assumptions made in the directed acyclic graph) $[28,29]$. The following known or possible breast cancer risk factors were considered 
as potential confounders and were included in the causal diagram: ethnicity (based on selfreported ethnicity in the CBCS and country of birth in the BCEES), education, family history of breast cancer, age at menarche, number of births, age at first birth, breastfeeding, use of oral contraceptives, use of hormone therapy, smoking status, alcohol consumption, recreational physical activity in early adulthood, recent recreational physical activity, body mass index in early adulthood, recent body mass index, total duration of employment, ever worked shift work, and years worked in an active occupation (Supplementary Figure 2). Based on the assumptions made in the directed acyclic graph, the following variables were considered to comprise an optimal sufficient set of confounders: education, ethnicity, recreational physical activity in early adulthood, body mass index in early adulthood, number of births, breastfeeding status, shift work status, and years worked in an active occupation. In sensitivity analyses, smoking status and alcohol consumption were added to the final pooled models, and their inclusion did not change the observed risk estimates. Trend tests were conducted by including the relevant ordinal variable into the model as a continuous variable. Tests for effect modification by menopausal status, country and lifetime recreational physical activity level were conducted by entering exposure-subgroup interaction terms in each model. We also stratified the pooled analyses by lifetime recreational physical activity to determine if the association between sedentary work and breast cancer risk was modified by lifetime recreational physical activity level. All analyses were performed using Stata 14.0 (StataCorp, College Station TX).

\section{RESULTS}

The characteristics of the participants are shown in Table 1, while Supplementary Table 2 shows these characteristics for pre-menopausal and post-menopausal women separately. Compared with participants in the BCEES, participants in the CBCS were more 
likely to be slightly younger, pre-menopausal, born in an Asian country, have a University degree, and to be nulliparous, and less likely to be overweight or obese at the time of study participation and in early adulthood, and less likely to be in the lowest quartile of lifetime recreational physical activity. In the pooled sample, cases were more likely than controls to have been born in an Asian country.

\section{Occupational characteristics}

The five most common occupational groups (seen in Supplementary Table 3) were office clerks and managers, retail salespersons/cashiers, nurses, secretaries and administrative assistants, bookkeeping and accounting clerks, and waiters and bar staff. In the BCEES, 44\% of the reported 16,988 jobs were self-rated as sedentary, 38\% as standing, and $17 \%$ as manual or heavy manual (Supplementary Table 3). In the CBCS, of the reported 10,326 jobs, 23\% were self-rated as sedentary, $37 \%$ as light, and $40 \%$ as moderate or heavy occupational physical activity. Participants in the BCEES were more likely to report a lower level of occupational physical activity than participants in the CBCS across job types (Supplementary Table 3).

Ratings of sedentary were estimated for $28 \%$ of jobs in the CBCS and for $39 \%$ of jobs in BCEES, using the Sit-JEM75, with $30 \%$ and $21 \%$ as standing, and $31 \%$ and $40 \%$ as active, in these two studies, respectively. There was moderate agreement between the Sit-JEM75 and self-reported occupational physical activity in the BCEES (linear weighted kappa = 0.54), and the CBCS (linear weighted kappa $=0.38$ ). The average Sit-JEM, Stand-JEM and WalkJEM values among jobs self-rated as sedentary was very similar across the two studies, but among jobs self-rated as standing/light, the average Sit-JEM value was higher in the CBCS 
than in the BCEES and the average Stand-JEM and Walk-JEM values were lower (Supplementary Table 4).

\section{Self-reported sedentary work and breast cancer risk}

In pooled analyses self-reported sedentary work was not associated with breast cancer risk among pre-menopausal women nor among post-menopausal women. In analysis of BCEES only, sedentary work was not associated with breast cancer risk for both menopausal groups. In the CBCS analysis, sedentary work was associated with elevated breast cancer risk among pre-menopausal women among those who had worked for 15 or more years in a sedentary job compared with those who had not worked in a sedentary occupation (OR=1.51, 95\% CI $\left.=0.91-2.51 ; P_{\text {Trend }}=0.037\right)$ (Table 2).

\section{Job-exposure matrix-assessed sedentary work and breast cancer risk}

In pooled analyses, sedentary work assessed through the Sit-JEM75 was not associated with the risk of pre-menopausal breast cancer, while it was associated with a nonsignificant decreased risk of post-menopausal breast cancer (OR for $15+$ years for 0 years $=$ 0.84, 95\% CI $\left.=0.67,1.05 ; P_{\text {Trend }}=0.072\right)$ (Table 2). In study-specific analyses, in the BCEES sedentary work assessed by Sit-JEM75 was associated with a non-significant decreased risk of breast cancer among post-menopausal women in the BCEES $\left(P_{\text {Trend }}=0.053\right)$; however, this association was not seen among postmenopausal women in the CBCS, nor among premenopausal women in either study. No association was observed in the pooled or studyspecific analyses for pre-menopausal or post-menopausal women when sedentary work was assessed using the SitJEM90, where a more strict definition of sedentary work was used. No effect modification was apparent by country, menopausal status, or lifetime recreational physical activity level. The results of the lifetime recreational physical activity-stratified 
pooled analyses are seen in Supplementary Table 5.

\section{DISCUSSION}

Self-reported sedentary work was associated with increased risk of breast cancer among pre-menopausal women in the CBCS; however, no such association was observed for JEM-assessed sedentary work, or in the BCEES separately. In the pooled analysis, we did not find consistent evidence that sedentary work was associated with the risk of breast cancer. When we used a more strict definition of sedentary jobs, we observed consistent null associations in both the pooled and study-specific analyses for pre-menopausal and postmenopausal women.

The results of previous research do not provide strong evidence that sedentary work is associated with breast cancer risk. A 2014 meta-analysis that examined the association between occupational sitting time and breast cancer reported a summary risk estimate of 1.03 for the highest versus lowest levels of sedentary work, and three subsequent studies (two cohort and one case-control) have also found no association between sitting time at work, total sitting time and/or time spent sitting watching television and the risk of breast cancer [12, 30, 31]. An updated meta-analysis published in 2015 found that occupational sedentary behaviour was associated with a $10 \%$ increased risk of breast cancer [10]. Thus, research to date indicates that occupational sedentary behaviour is unlikely to have a large influence on overall breast cancer risk.

Although research indicates risk factors related to sedentary behaviour, such as obesity and physical activity, may have differential associations with breast cancer risk depending on menopausal status [11], the results of this study and some previous studies do not provide strong evidence that sedentary behaviour is differentially associated with breast cancer risk among pre-menopausal and post-menopausal women. Six previous studies have 
examined whether sedentary behaviour is differentially associated with the risks of pre- and post-menopausal breast cancer [12-17]. Three found no association between sedentary behaviour and pre- or post-menopausal breast cancer [12, 13, 16], one reported increased risk for overall breast cancer and no difference based on menopausal status [17], one study found increased risk for post-menopausal breast cancer but no association for pre-menopausal breast cancer [14], and finally, one study found decreased risk for post-menopausal breast cancer and a slightly weaker decreased risk for pre-menopausal breast cancer [15]. Four studies conducted exclusively among post-menopausal women found no association between sitting time and breast cancer risk [32-35], while another study reported increased risk among post-menopausal women [36].

This analysis has several strengths and limitations. The large sample size obtained by pooling two studies allowed us to examine the associations between sedentary work and breast cancer in pre-menopausal and post-menopausal women separately. The two studies were similar in several ways: they were conducted in overlapping time periods; both asked participants to recall the physical activity requirements of their occupations over the lifetime and their lifetime recreational and household physical activity; and both collected similar information about other demographic, lifestyle-related, reproductive and occupational breast cancer risk factors. However, neither study collected information about sedentary behaviour in non-occupational domains, such as in leisure-time (e.g., watching television) or transportrelated sedentary behaviour, so were not able to consider the influence of sitting in these domains (or total sitting time) on breast cancer risk. Both the self-reported and JEM-based measures of occupational physical activity used in this study have limitations, and the ability of the self-reported and JEM-based measures to collect valid and reliable information about occupational activity over the lifetime is not known. The self-report measures did not allow 
participants to report multiple job tasks requiring different levels of activity, and did not provide information about sitting duration. JEM-based measures also have several limitations [37]: for example, they assume that people working in the same job have the same occupational physical activity level, which is not necessarily the case. The limitations of these methods mean exposure misclassification was present in this study. This misclassification is likely to have been non-differential, so may have biased the observed results towards the null. Further research is needed to determine whether self-reported and/or JEM-based methods can measure actual sedentary work exposures with acceptable accuracy. A further limitation is that self-reported ethnicity was not collected in the BCEES, so country of birth was used as a proxy measure. This is unlikely to have resulted in misclassification of ethnicity however, since based on Census data the concordance between country of birth and self-reported ancestry in Western Australian females is very high [38].

It appeared participants in the two studies rated their occupational activity differently. Specifically, participants in the CBCS rated their occupational activity as more active than participants in the BCEES), There could be three explanations for this difference. First, participants in the two studies may have been working in different kinds of jobs, but the distribution of job-type was roughly equivalent in the two studies so this explanation is unlikely. Second, the same job may require different levels of sitting and/or physical activity in the two countries. While this may be possible for particular occupations, we found large differences in the distribution of self-reported occupational physical activity across all job types. Finally, differences in the questions used to collect occupational activity in the two studies may have resulted in participants rating their occupational physical activity differently. Although both studies labelled the least active category 'sedentary', the definition of sedentary jobs in the BCEES were those where you spend "most of your time sitting," 
while in the CBCS they were defined as jobs that require "sitting only, with minimal walking.” The second least active category had a similar definition in the two studies, but a different label: 'standing' in the BCEES, and 'light' in the CBCS. The use of example jobs for the different intensity levels in the BCEES, but not in the CBCS, may also have contributed to the difference. These findings imply that small differences in the way a question is worded can have a large impact on the data that are subsequently collected, as previous research has reported [39, 40].

In summary, we observed increased breast cancer risk associated with self-reported sedentary behaviour among pre-menopausal women in the Canadian study; however, in pooled analyses with a large study in Australia, we did not find evidence that sedentary work is associated with the risk of breast cancer.

\section{Competing interests: None declared}

Acknowledgements: The Breast Cancer Environment and Employment Study was supported by the Australian National Health and Medical Research Council (NHMRC Grant \#572530) and by a grant from the Cancer Council Western Australia. The Canadian Breast Cancer Study was supported by the Canadian Institutes of Health Research (Grant \#69036). Terry Boyle is supported by the Canadian Institutes of Health Research (Fellowship \#300068), the Michael Smith Foundation for Health Research (Postdoctoral Fellowship \#5553), Killam Trusts (Honorary Postdoctoral Research Fellowship), and the NHMRC (Early Career Fellowship \#1072266). Lin Fritschi is supported by the NHMRC (Fellowship \#37614900) and Cancer Council Western Australia (Fellowship). The authors acknowledge and thank all staff and students who contributed to the BCEES and the CBCS, particularly Zenaida 
Abonto, Agnes Lai and Anne Grundy for their help with this study. The authors declare that they have no conflict of interest.

Ethical approval: All procedures performed in studies involving human participants were in accordance with the ethical standards of the institutional and/or national research committee and with the 1964 Helsinki declaration and its later amendments or comparable ethical standards. The studies reported in this manuscript comply with the current laws of the country in which they were performed.

Contributorship Statement: TB drafted the manuscript, prepared, harmonized and pooled the data, and conducted all statistical analyses and data interpretation. LF, LCK, JSH, DGL, SS, KJA and JJS contributed to data preparation, data analysis and/or interpretation of data, and provided feedback on the original and revised drafts of the manuscript. LF, JSH, KJA and JJS were also involved in the conception and design of the individual studies. All authors have read and approved of the submitted manuscript. 
Table 1: Descriptive statistics of cases and controls in the Breast Cancer Environment and Employment Study (BCEES) and the Canadian Breast Cancer Study (CBCS)

\begin{tabular}{|c|c|c|c|c|c|c|}
\hline & Pooled & & BCEES & & CBCS & \\
\hline & $\begin{array}{l}\text { Controls } \\
(n=2532)\end{array}$ & $\begin{array}{l}\text { Cases } \\
(n=1762)\end{array}$ & $\begin{array}{l}\text { Controls } \\
(n=1452)\end{array}$ & $\begin{array}{l}\text { Cases } \\
(n=940)\end{array}$ & $\begin{array}{l}\text { Controls } \\
(n=1080)\end{array}$ & $\begin{array}{l}\text { Cases } \\
(n=822)\end{array}$ \\
\hline Characteristic & $\%$ & $\%$ & $\%$ & $\%$ & $\%$ & $\%$ \\
\hline \multicolumn{7}{|l|}{ Age group } \\
\hline 40 to 49 years & 23.3 & 27.8 & 18.7 & 25.6 & 29.4 & 30.2 \\
\hline 50 to 59 years & 33.6 & 32.0 & 32.6 & 30.4 & 35.1 & 33.8 \\
\hline 60 to 69 years & 30.0 & 26.6 & 34.5 & 30.3 & 24.0 & 22.3 \\
\hline $70+$ years & 13.1 & 13.7 & 14.2 & 13.6 & 11.6 & 13.7 \\
\hline \multicolumn{7}{|l|}{ Ethnicity } \\
\hline White & 87.0 & 79.9 & 93.6 & 92.1 & 78.2 & 65.8 \\
\hline Asian & 9.4 & 16.2 & 3.6 & 4.5 & 17.3 & 29.7 \\
\hline Other & 3.5 & 3.9 & 2.8 & 3.4 & 4.4 & 4.5 \\
\hline \multicolumn{7}{|l|}{ Education } \\
\hline Did not complete high school & 22.7 & 24.3 & 35.5 & 35.6 & 5.6 & 11.3 \\
\hline Completed high school & 21.1 & 20.3 & 22.3 & 20.2 & 19.4 & 20.4 \\
\hline Trade/apprenticeship/diploma & 26.7 & 26.3 & 24.8 & 21.8 & 29.3 & 31.4 \\
\hline University degree & 29.5 & 29.1 & 17.5 & 22.3 & 45.7 & 36.9 \\
\hline \multicolumn{7}{|l|}{ Number of births } \\
\hline 0 & 17.9 & 17.5 & 9.7 & 11.4 & 28.9 & 24.6 \\
\hline 1 & 9.4 & 13.3 & 6.8 & 9.7 & 12.8 & 17.4 \\
\hline 2 & 37.8 & 37.6 & 39.3 & 39.9 & 35.7 & 35.0 \\
\hline $3+$ & 35.0 & 31.6 & 44.2 & 39.0 & 22.6 & 23.0 \\
\hline \multicolumn{7}{|l|}{ Ever breastfed (all participants) } \\
\hline No & 28.4 & 30.8 & 18.0 & 21.6 & 42.3 & 41.4 \\
\hline Yes & 71.6 & 69.2 & 82.0 & 78.4 & 57.7 & 58.6 \\
\hline \multicolumn{7}{|c|}{ Ever breastfed (participants with 1+ births) } \\
\hline No & 12.7 & 16.1 & 9.2 & 11.5 & 18.9 & 22.3 \\
\hline Yes & 87.3 & 83.9 & 90.8 & 88.5 & 81.1 & 77.7 \\
\hline \multicolumn{7}{|l|}{ Menopausal status } \\
\hline Post-menopausal & 70.3 & 66.1 & 78.9 & 71.9 & 58.6 & 59.4 \\
\hline Pre-menopausal & 29.7 & 33.9 & 21.1 & 28.1 & 41.4 & 40.6 \\
\hline \multicolumn{7}{|l|}{ Current body mass index } \\
\hline Underweight/Normal weight & 51.1 & 49.9 & 44.8 & 45.0 & 59.5 & 54.3 \\
\hline Overweight & 30.7 & 32.1 & 33.4 & 33.2 & 27.2 & 30.9 \\
\hline Obese & 18.2 & 18.6 & 21.9 & 21.9 & 13.2 & 14.8 \\
\hline \multicolumn{7}{|l|}{ Early adulthood body mass index } \\
\hline Underweight/Normal weight & 85.1 & 86.8 & 80.8 & 82.1 & 90.8 & 92.2 \\
\hline Overweight & 11.3 & 9.8 & 14.4 & 12.8 & 7.0 & 6.4 \\
\hline Obese & 3.7 & 3.3 & 4.8 & 5.1 & 2.1 & 1.3 \\
\hline
\end{tabular}




\begin{tabular}{|c|c|c|c|c|c|c|}
\hline \multicolumn{7}{|l|}{ Lifetime recreational physical activity } \\
\hline 0 to 5.9 MET-hours/week & 25.6 & 31.0 & 21.6 & 22.7 & 30.9 & 40.6 \\
\hline 6 to 13.9 MET-hours/week & 24.4 & 22.8 & 26.6 & 22.8 & 21.6 & 22.7 \\
\hline 14 to 27.9 MET-hours/week & 25.7 & 24.2 & 29.1 & 28.2 & 21.0 & 19.6 \\
\hline 28+ MET-hours/week & 24.3 & 22.0 & 22.7 & 26.4 & 26.5 & 17.0 \\
\hline \multicolumn{7}{|c|}{ Early adulthood recreational physical activity } \\
\hline 0 to 9.9 MET-hours/week & 46.4 & 47.0 & 46.8 & 44.0 & 45.7 & 50.3 \\
\hline 10 to 29.9 MET-hours/week & 25.9 & 26.0 & 27.4 & 26.7 & 24.0 & 25.2 \\
\hline 30+ MET-hours/week & 27.7 & 27.0 & 25.8 & 29.3 & 30.3 & 24.5 \\
\hline \multicolumn{7}{|l|}{ Total time worked } \\
\hline 0 to 15.9 years & 24.1 & 24.7 & 26.2 & 28.0 & 21.4 & 21.0 \\
\hline 16 to 23.9 years & 25.6 & 25.3 & 26.0 & 27.2 & 24.9 & 23.0 \\
\hline 24 to 31.9 years & 25.6 & 24.2 & 26.0 & 22.3 & 24.9 & 26.4 \\
\hline $32+$ years & 24.8 & 25.8 & 21.8 & 22.4 & 28.8 & 29.6 \\
\hline \multicolumn{7}{|l|}{ Ever worked shiftwork } \\
\hline No & 63.2 & 60.7 & 62.1 & 58.2 & 64.7 & 63.6 \\
\hline Yes & 36.8 & 39.3 & 37.8 & 41.8 & 35.3 & 36.4 \\
\hline \multicolumn{7}{|l|}{ Self-reported sedentary work } \\
\hline 0 years & 40.6 & 44.2 & 25.9 & 28.4 & 60.5 & 62.2 \\
\hline 0.1 to 6.99 years & 19.0 & 18.9 & 23.8 & 23.6 & 12.6 & 13.5 \\
\hline 7 to 18.49 years & 20.3 & 18.3 & 25.3 & 24.0 & 13.6 & 11.7 \\
\hline $18.5+$ years & 20.0 & 18.7 & 25.0 & 23.9 & 13.3 & 12.7 \\
\hline \multicolumn{7}{|l|}{ Sit-JEM75-based sedentary work ${ }^{\mathrm{a}}$} \\
\hline 0 years & 31.6 & 33.8 & 29.3 & 33.0 & 34.7 & 34.7 \\
\hline 0.1 to 6.99 years & 22.1 & 23.3 & 25.6 & 27.4 & 17.4 & 18.6 \\
\hline 7 to 18.49 years & 23.1 & 22.5 & 24.4 & 21.5 & 21.2 & 23.6 \\
\hline $18.5+$ years & 23.3 & 20.4 & 20.7 & 18.1 & 26.7 & 23.1 \\
\hline \multicolumn{7}{|l|}{ Sit-JEM90-based sedentary work ${ }^{\mathrm{b}}$} \\
\hline 0 years & 71.2 & 72.4 & 66.0 & 66.9 & 78.1 & 78.7 \\
\hline 0.1 to 2.99 years & 9.5 & 9.4 & 10.4 & 11.3 & 8.3 & 7.2 \\
\hline 3 to 6.99 years & 9.8 & 8.6 & 12.8 & 10.6 & 5.8 & 6.3 \\
\hline $7+$ years & 9.5 & 9.6 & 10.8 & 11.2 & 7.7 & 7.8 \\
\hline \multicolumn{7}{|l|}{ Self-reported active work } \\
\hline No active work (\%) & 50.6 & 45.0 & 62.1 & 56.8 & 35.1 & 31.5 \\
\hline Mean years in active work (SD) & $7.1 \mathrm{yrs}(12.0)$ & 8.1 yrs (12.6) & 4.2 yrs (8.6) & 4.6 yrs $(9.2)$ & 11.0 yrs (14.4) & 12.2 yrs (14.6) \\
\hline \multicolumn{7}{|l|}{ Sit-JEM75/90-based active work ${ }^{\mathrm{c}}$} \\
\hline No active work (\%) & 33.4 & 31.2 & 27.1 & 25.2 & 41.9 & 38.0 \\
\hline Mean years in active work (SD) & 7.4 yrs (10.5) & 8.0 yrs (11.4) & 8.4 yrs (10.7) & 8.6 yrs (10.9) & 6.2 yrs (10.0) & 7.4 yrs (11.9) \\
\hline
\end{tabular}

Abbreviations: BCEES, Breast Cancer Environment and Employment Study; CBCS, Canadian Breast Cancer Study; JEM, Job Exposure matrix; MET, Metabolic equivalent; yrs, Years.

a job with a value of 75 or greater in the 'Occupational Information Network 'Work Context - Spend Time Sitting' job-exposure matrix

${ }^{b}$ A job with a value of 90 or greater in the 'Occupational Information Network 'Work Context - Spend Time Sitting' jobexposure matrix

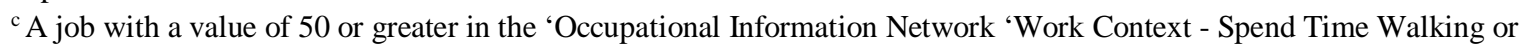
Running' job-exposure matrix 
Table 2: Association between sedentary work and the risk of breast cancer in the Breast Cancer Environment and Employment Study (BCEES) and the Canadian Breast Cancer Study (CBCS) by menopausal status, overall and by study

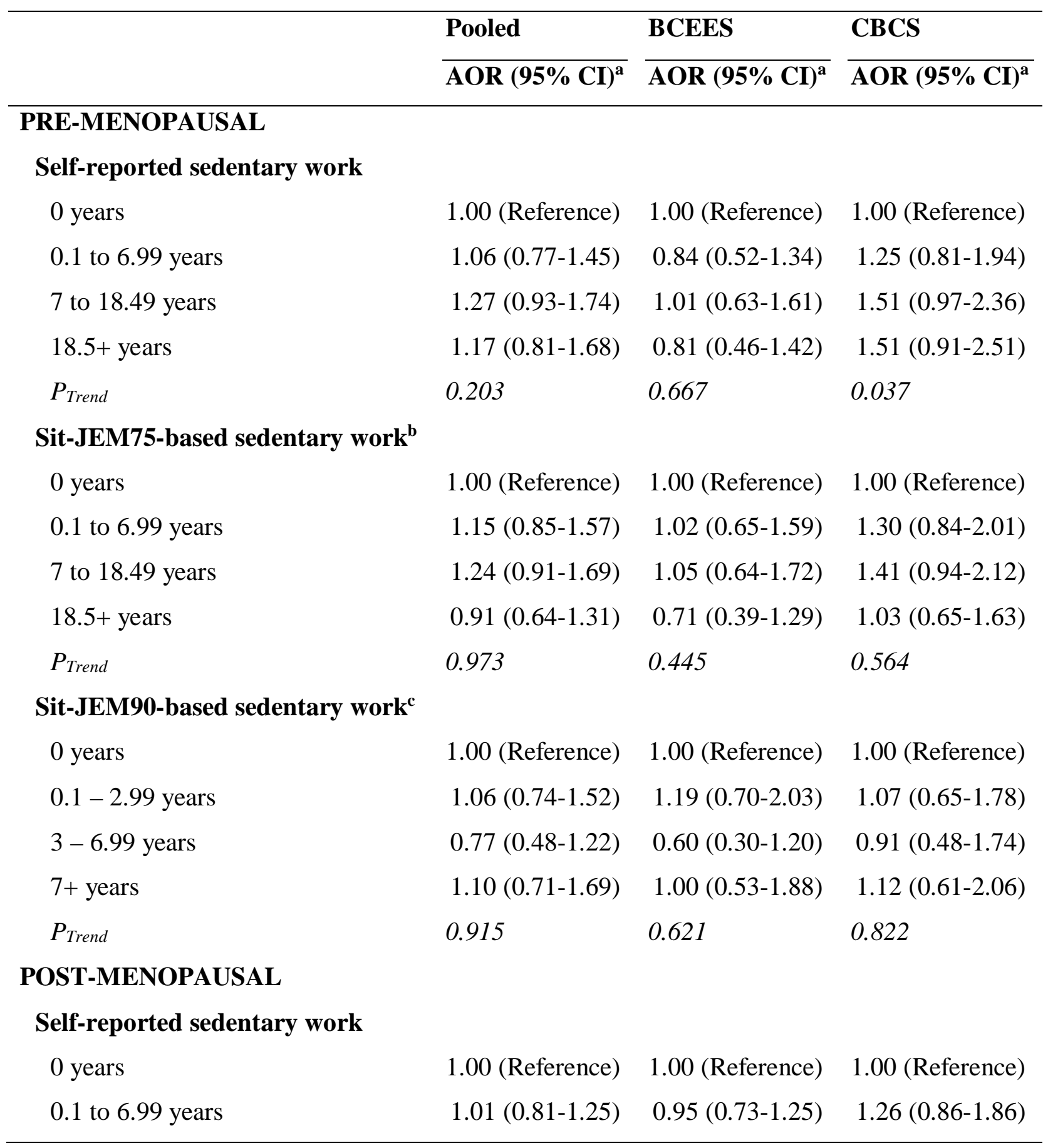




\begin{tabular}{llll}
\hline 7 to 18.49 years & $0.80(0.64-1.01)$ & $0.87(0.66-1.16)$ & $0.63(0.41-0.97)$ \\
18.5+ years & $0.90(0.72-1.12)$ & $0.91(0.69-1.21)$ & $0.86(0.59-1.26)$ \\
$P_{\text {Trend }}$ & 0.150 & 0.431 & 0.189 \\
Sit-JEM75-based sedentary work & & & \\
0 years & & & \\
0.1 to 6.99 years & 1.00 (Reference) & 1.00 (Reference) & 1.00 (Reference) \\
7 to 18.49 years & $1.02(0.83-1.26)$ & $0.94(0.72-1.21)$ & $1.19(0.82-1.72)$ \\
$18.5+$ years & $0.85(0.68-1.06)$ & $0.75(0.56-0.99)$ & $1.05(0.73-1.51)$ \\
$P_{\text {Trend }}$ & $0.84(0.67-1.05)$ & $0.78(0.58-1.07)$ & $0.90(0.64-1.27)$ \\
Sit-JEM90-based sedentary work & \\
0 years & 0.072 & 0.053 & 0.500 \\
$0.1-2.99$ years & $1.00($ Reference) & $1.00($ Reference) & 1.00 (Reference) \\
3 - 6.99 years & $1.05(0.80-1.38)$ & $1.11(0.81-1.54)$ & $0.97(0.58-1.62)$ \\
$7+$ years & $1.04(0.80-1.34)$ & $0.98(0.72-1.33)$ & $1.42(0.85-2.38)$ \\
$P_{\text {Trend }}$ & $1.06(0.82-1.36)$ & $1.13(0.82-1.55)$ & $0.94(0.60-1.47)$ \\
\hline
\end{tabular}

Abbreviations: AOR, Adjusted Odds Ratio; BCEES, Breast Cancer Environment and Employment Study; CBCS, Canadian Breast Cancer Study; CI, Confidence Interval; JEM, Job Exposure matrix.

${ }^{a}$ Adjusted for age, study location, education, ethnicity, recreational physical activity in early adulthood, body mass index in early adulthood, number of births, breastfeeding status, shift work status, and years worked in an active occupation.

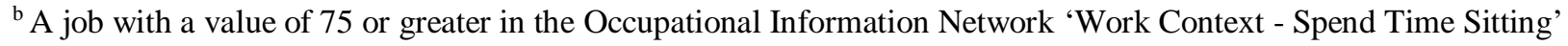
job-exposure matrix

' A job with a value of 90 or greater in the Occupational Information Network 'Work Context - Spend Time Sitting' job-exposure matrix 


\section{REFERENCES}

1 Lynch BM, Neilson HK, Friedenreich CM. Physical activity and breast cancer prevention. Recent Results Cancer Res 2011;186:13-42.

2 Sedentary Behaviour Research Network. Letter to the Editor: Standardized use of the terms “sedentary" and "sedentary behaviours”. Appl Physiol Nutr Metab 2012;37(3):540-42.

3 Biswas A, Oh PI, Faulkner GE, et al. Sedentary time and its association with risk for disease incidence, mortality, and hospitalization in adults: a systematic review and meta-analysis. Ann Intern Med 2015;162(2):123-32.

4 Schmid D, Leitzmann MF. Television viewing and time spent sedentary in relation to cancer risk: a meta-analysis. J Natl Cancer Inst 2014;106(7).

5 van Uffelen JGZ, Wong J, Chau JY, et al. Occupational sitting and health risks: a systematic review. Am J Prev Med 2010;39(4):379-88.

6 Ferlay J, Soerjomataram I, Ervik M, et al. GLOBOCAN 2012 v1.0, Cancer Incidence and Mortality Worldwide: IARC CancerBase No. 11 [Internet]. Secondary GLOBOCAN 2012 v1.0, Cancer Incidence and Mortality Worldwide: IARC CancerBase No. 11 [Internet] 2013. http://globocan.iarc.fr.

7 World Health Organisation / World Economic Forum. Preventing noncommunicable diseases in the workplace through diet and physical activity: WHO/World Economic Forum report of a joint event. Geneva, Switzerland: World Health Organisation / World Economic Forum, 2008.

8 Lynch BM. Sedentary behavior and cancer: a systematic review of the literature and proposed biologic mechanisms. Cancer Epidemiol Biomarkers Prev 2010;19(11):2691-709.

9 Shen D, Mao W, Liu T, et al. Sedentary behavior and incident cancer: a meta-analysis of prospective studies. PLoS ONE 2014;9(8):e105709.

10 Zhou Y, Zhao H, Peng C. Association of sedentary behavior with the risk of breast cancer in women: update meta-analysis of observational studies. Ann Epidemiol 2015.

11 World Cancer Research Fund, American Institute for Cancer Research. Continuous Update Project report. Food, nutrition, physical activity, and the prevention of breast cancer. Washington DC: AICR, 2010. 
12 Catsburg C, Kirsh V, Soskolne C, et al. Associations between anthropometric characteristics, physical activity, and breast cancer risk in a Canadian cohort. Breast Cancer Res Treat 2014;145(2):545-52.

13 Coogan PF, Newcomb PA, Clapp RW, et al. Physical activity in usual occupation and risk of breast cancer (United States). Cancer Causes Control 1997;8(4):626-31.

14 Kruk J. Lifetime occupational physical activity and the risk of breast cancer: a case-control study. Asian Pac J Cancer Prev 2009;10(3):443-8.

15 Lynch B, Courneya K, Friedenreich C. A case-control study of lifetime occupational sitting and likelihood of breast cancer. Cancer Causes Control 2013;24(6):1257-62.

16 Mathew A, Gajalakshmi V, Rajan B, et al. Physical activity levels among urban and rural women in south India and the risk of breast cancer: a case-control study. Eur J Cancer Prev 2009;18(5):368-76.

17 Pronk A, Ji BT, Shu XO, et al. Physical activity and breast cancer risk in Chinese women. $\mathrm{Br}$ J Cancer 2011;105(9):1443-50.

18 Si S, Boyle T, Heyworth J, et al. Lifetime physical activity and risk of breast cancer in preand post-menopausal women. Breast Cancer Res Treat 2015;152(2):449-62.

19 Kobayashi L, Janssen I, Richardson H, et al. Moderate-to-vigorous intensity physical activity across the life course and risk of pre- and post-menopausal breast cancer. Breast Cancer Res Treat 2013;139(3):851-61.

20 Kobayashi L, Janssen I, Richardson H, et al. A case-control study of lifetime light intensity physical activity and breast cancer risk. Cancer Causes Control 2014;25(1):133-40.

21 Fritschi L, Erren TC, Glass DC, et al. The association between different night shiftwork factors and breast cancer: a case-control study. Br J Cancer 2013;109(9):2472-80.

22 Cust A, Smith B, Chau J, et al. Validity and repeatability of the EPIC physical activity questionnaire: a validation study using accelerometers as an objective measure. Int $J$ Behav Nutr Phys Act 2008;5(1):33.

23 Friedenreich CM, Courneya KS, Bryant HE. The Lifetime Total Physical Activity Questionnaire: development and reliability. Med Sci Sports Exerc 1998;30(2):266-74.

24 U.S. Department of Labor. Dictionary of Occupational Titles 4th ed. Washington, DC: US Government Printing Office 1991. 
25 Boyle T, Leong S. Comparing ratings of occupational physical activity. Epidemiology 2012;23(6):934-36.

26 National Center for O*NET Development. O*NET Resource Center. Secondary O*NET Resource Center 2015. https://www.onetcenter.org/.

27 National Center for O*NET Development. O*NET® 19.0 Database. Raleigh, North Carolina: National Center for O*NET Development, 2014. Accessed March 2015. https://www.onetcenter.org/database.html.

28 Textor J, Hardt J, Knüppel S. DAGitty: A graphical tool for analyzing causal diagrams. Epidemiology 2011;22(5):745.

29 Greenland S, Pearl J, Robins JM. Causal diagrams for epidemiologic research. Epidemiology 1999;10(1):37-48.

30 Rosenberg L, Palmer JR, Bethea TN, et al. A prospective study of physical activity and breast cancer incidence in African-American women. Cancer Epidemiol Biomarkers Prev 2014;23(11):2522-31.

31 Ekenga C, Parks C, Sandler D. A prospective study of occupational physical activity and breast cancer risk. Cancer Causes Control 2015;26:1779-89.

32 Hildebrand JS, Gapstur SM, Campbell PT, et al. Recreational physical activity and leisuretime sitting in relation to postmenopausal breast cancer risk. Cancer Epidemiol Biomarkers Prev 2013;22(10):1906-12.

33 George SM, Irwin ML, Matthews CE, et al. Beyond recreational physical activity: examining occupational and household activity, transportation activity, and sedentary behavior in relation to postmenopausal breast cancer risk. Am J Public Health 2010;100(11):2288-95.

34 Dirx MJM, Voorrips LE, Goldbohm RA, van den Brandt PA. Baseline recreational physical activity, history of sports participation, and postmenopausal breast carcinoma risk in the Netherlands Cohort Study. Cancer 2001;92(6):1638-49.

35 Moradi T, Nyren O, Zack M, et al. Breast cancer risk and lifetime leisure-time and occupational physical activity (Sweden). Cancer Causes Control 2000;11(6):523-31.

36 Cohen SS, Matthews CE, Bradshaw PT, et al. Sedentary behavior, physical activity, and likelihood of breast cancer among Black and White women: a report from the Southern Community Cohort Study. Cancer Prev Res 2013;6(6):566-76. 
37 Lynch BM, Boyle T. Distinguishing sedentary from inactive: implications for meta-analyses. Br J Cancer 2014;111(11):2202-03.

38 Australian Bureau of Statistics. Table generated with ABS Table Builder using 2011 Census of Population and Housing data. Canberra, Australia: Australian Bureau of Statistics, 2011. Accessed June 2016.

http://www.abs.gov.au/websitedbs/censushome.nsf/home/tablebuilder.

39 Uitenbroek D, McQueen D. Leisure time physical activity in Scotland: Trends 1987-1991 and the effect of question wording. Soz Praventivmed 1992;37(3):113-17.

40 Jørgensen ME, Sørensen MR, Ekholm O, Rasmussen NK. Importance of questionnaire context for a physical activity question. Scand J Med Sci Sports 2013;23(5):651-56. 